[recto rh: Process Simulation During the Design Process]

\title{
Process Simulation during the Design Process Makes the Difference: Process Simulations Applied to a Traditional Design
}

Roberto Traversari, BSc, MBA; Rien Goedhart, MSc; and Jan Maarten Schraagen, PhD

[CEU burst]

$<$ abstract $>$

Objective: The objective is evaluation of a traditionally designed operating room using simulation of various surgical workflows.

Background: A literature search showed that there is no evidence for an optimal operating room layout regarding the position and size of an ultraclean ventilation (UCV) canopy with a separate preparation room for laying out instruments and in which patients are induced in the operating room itself. Neither was literature found reporting on process simulation being used for this application. Many technical guidelines and designs have mainly evolved over time, and there is no evidence on whether the proposed measures are also effective for the optimization of the layout for workflows.

Methods: The study was conducted by applying observational techniques to simulated typical surgical procedures. Process simulations which included complete surgical teams and equipment required for the intervention were carried out for four typical interventions. Four observers used a form to record conflicts with the clean area boundaries and the height of the supply bridge. Preferences for particular layouts were discussed with the surgical team after each simulated procedure.

Results: We established that a clean area measuring 3 x 3 meters and a supply bridge height of 2.05 meters was satisfactory for most situations, provided a movable operation table is used. The only cases in which conflicts with the supply bridge were observed were during the use of a surgical robot (Da Vinci) and a surgical microscope. During multiple trauma interventions, bottlenecks regarding the dimensions of the clean area will probably arise.

Conclusions: The process simulation of four typical interventions has led to significantly different operating room layouts than were arrived at through the traditional design process. 
Keywords: Architecture, Methodology, Evidence-based design, Human factors, Work environment, Operating room Traditional design, Process simulation, , Surgical workflow, ) $</$ abstract $>$

Author Affiliations: Mr. Traversari is a senior researcher advisor at The Netherlands Organisation for Applied Scientific Research TNO in The Netherlands. Mr. Goedhart is an adviser at the Building Department of Erasmus MC, University Medical Center Rotterdam. Mr. Jan Maarten Schraagen is a senior researcher at The Netherlands Organisation for Applied Scientific Research TNO in The Netherlands.

Corresponding Author: Roberto Traversari, BSc, MBA, Dutch Centre for Health Assets (DuCHA), The Netherlands Organisation for Applied Scientific Research TNO, Kampweg 5, 3769 DE Soesterberg, The Netherlands (roberto.traversari@tno.nl).

Acknowledgments: The authors thank the participating surgeons, nurses, and staff of the operating department of Erasmus MC and the observers who participated in this project. They also thank the many readers from Erasmus MC and TNO who commented on the concept article to make it better. In addition they thank Erasmus MC for facilitating the project and the Dutch Ministry of Health, Welfare and Sport for funding the research performed by TNO.

Preferred Citation: Traversari, R., Goedhart, R., \& Schraagen, J. M. (2012). Process simulation during the design process makes the difference: Process simulations applied to a traditional design. Health Environments Research \& Design Journal 6(2), [pp tk].

\section{Introduction}

The objective of this study is to evaluate a traditionally designed operating room using simulation of various surgical workflows. The following hypotheses were tested during the evaluation process:

- The $3 \times 3 \mathrm{~m}^{2}$ clean area surface is large enough to carry out all interventions within it.

- Positioning the clean area in the middle of the operating room results in an optimal layout for moving in the patient and equipment in the operating room.

- The classic position of the anesthesia section, near the entrance to the operating room, is optimal for the logistical processes. 
- A supply bridge height of 2.05 meters results in the clean area being sufficiently accessible for high medical equipment and reachable for the staff to make connections (electricity, vacuum, etc.).

\section{Background}

A traditional design process is defined as one in which the architect draws a layout based on the descriptions of operating room processes he or she receives (Van Boxel, et al., 2007). The main characteristics of a traditional design process are that it makes use of the experiences and insights of the parties involved, without subjecting the designed situation to functionality testing using simulation prior to the actual construction of the design (Van Boxel et al., 2007). Although there is a discernible trend towards an increased use of evidence-based design of healthcare facilities (Gawron, Dennison, \& Biferno, 2002; Reiling et al., 2004; Sadler et al., 2011; Sandberg, 2005; Dunston, Arns, \& McGlothlin, 2007; Bittermann, 2009), little evidence is available for the optimal layout of an operating room. We therefore carried out a literature review, starting with the comprehensive literature review carried out by Ulrich et al. (2008).

This review surveyed and evaluated evidence-based healthcare design and extracted its implications for the design of better and safer hospitals. This review was taken as a starting point in order to evaluate whether there were relevant studies on operating room layout-the purpose of this particular study. The literature reviewed regarding operating rooms concerned the following topics: (1) High-Efficiency Particulate Air (HEPA) Filters (Sehulster et al., 2004; Wickens, Lee, Liu, \& Gordon Becker, 2004); (2) Ventilation Systems and Airflow Control (Yavuz et al., 2006; Gawron et al., 2002); and (3) Reduce Staff Stress by Controlling Noise (Moorthy, Munz, Undre, \& Darzi, 2004; Sanderson et al., 2005). It can be concluded that no reference to literature that dealt with optimization of operating room layouts as proposed in this study was found in this review (Ulrich et al., 2008).

Next, a literature search was carried out using the following databases: Cochrane Library, PubMed, Scopus, ScienceDirect, and Google Scholar. The search included only literature published between 2004 and 2011, so only studies based on the actual contemporary situation were included. The following search terms or a combination of these terms were used: operation room, operating theatre/theater/room, lay-out/layout, spatial organization, 
optimization, design/configuration, surgical suite, process simulation. Several studies (17) published after 2008 relating to operating rooms and design can be found that used different research methods. In 2011, Chen et al. studied the clinical practicability of a dual-room 1.5-T intraoperative magnetic resonance imaging (iMRI) suite, and concluded that it easily could be integrated into standard neurosurgical workflow.

Strauss et al. (2010) compared the Ear, Nose, and Throat (ENT) operating room (OR) with the previous standard, including a surgical workflow analysis, and concluded that a contemporary operation unit can significantly improve safety and efficiency as well as the ergonomics for ENT surgery and related procedures. The concept of operating room zoning has also undergone a dramatic change. Some research suggests that a layout focusing on work efficiency is more desirable than one that follows an excessively rigid zoning pattern (Suzuki, 2010). In order to study the impact of facility change (e.g., from decentralized to centralized ORs) on OR processes, a group of researchers (Baumgart, Denz, Bender, \& Schleppers, 2009) used a mixed method approach (process analysis, process modeling and social network analysis of staff interaction) to analyze contextual factors (e.g., department layout) as well as behavioral patterns (e.g., staff interactions). According to this study, such a mixed method approach, as compared to a single assessment method, enabled a deeper understanding of the OR work context and its influence on outpatient OR processes, concluding that, for example, there is a potential for more errors during handovers in the new centralized design due to a greater interdependency between tasks and staff. This is a "before-after" type of study, where an old situation and a new situation are analyzed and conclusions are made according to the analysis. This study is a good example of the fact that simply analyzing what went wrong after a building is already in use has disadvantages. Various methods can be used to analyze the potential risks of design solutions before they are realized and implemented in a building on a large scale. Process simulation, as described in this paper, could be one of them. Such studies are highly beneficial because the results of these studies can be used to prevent some "obvious” design shortcomings in advance (Sadler et al., 2011).

Another method that can be used for design analysis during the design process is the use of virtual reality mock-ups (Dunston et al., 2007; Peavey et al., 2012). Virtual models are a good possibility but one needs to carefully consider the costs of building a virtual or physical 
model based on specific research requirements. Mock-ups enable developers to visualize and evaluate the physical interface while the design is still in progress. In order to study whether the expected range of users can optimally use the proposed design solution, or whether visibility is acceptable and maintainers have good access to the equipment, real time mockups could be used (Gawron et al., 2002). A group of authors (Sandberg et al, 2005) constructed a three-room suite including an OR, an induction room, and an early recovery area. The authors sought to improve OR throughput and reduce time per case by goaldirected design of a demonstration OR (which was constructed) and the perioperative processes occurring within and around it. The authors compared the throughput, cost, and revenue performance of the new OR to traditional ORs. The main conclusions from the study were that intentional OR and perioperative process redesign improved throughput. Better OR throughput entailed additional costs but allowed additional patients to be accommodated in the OR while generating revenue that balanced these additional costs.

In conclusion, the literature review showed that there have been only a few studies employing process simulation in the design of new operating rooms. Our study adds to this limited evidence base by simulating future OR layouts, carrying out process simulations with medical teams in these simulated layouts, and evaluating in advance user response to the proposed designs.

\section{Case Study}

The case study was carried out in a large academic medical teaching hospital in The Netherlands. The schedule of requirements for the new ORs in this hospital described the processes for the future OR in terms of current practices and the perception of the influence of other logistical and technical starting points on this process. The main functional requirements in our case were:

1. The choice to create a separate instrument preparation room connected to the operating room.

2. The choice to make use of standard unidirectional down-flow plenums measuring 3.0 $\mathrm{x} 3.0 \mathrm{~m}^{2}$ as is commonly applied in new buildings in The Netherlands.

3. The choice to make use of a fixed supply bridge with side screens around the plenum. 
These starting points were translated to a spatial “classic” layout by the architect (Figure 1), resulting in:

1. A spatially centered design of the plenum and, with it, the clean area in the operating room.

2. Positioning of the anesthesia system on the corridor side of the clean area, as is common practice in The Netherlands.

3. An entrance to the preparation room in the wall at right angles to the front. This entrance is situated near the front.

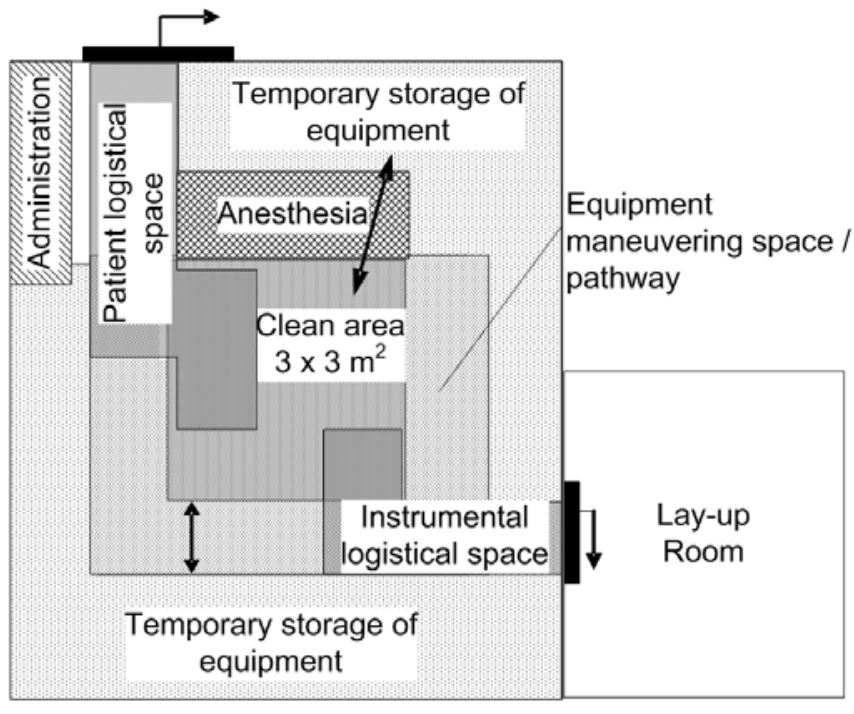

OR layout, classical

Figure 1: OR layout, result of the classical design process.

Some doubts on the solutions chosen remained among the future users of the OR because the starting points for the OR layout partly relate to techniques and logistical processes that are new for the hospital. The choice of clean area surface, in particular, in combination with the choice for a supply bridge, led to doubts on the feasibility of successfully positioning the surgical teams and equipment within the designated clean zone in the operating room. In particular, the research question was whether the clean area under the unidirectional air flow terminal would be large enough to enable smooth, unhindered, task performance, while at the same time not being too far away to reach it with the equipment connections. This research question led to the decision to construct a mock-up supply bridge and to make use of 
simulations to study whether the dimensions chosen in the design are satisfactory (Wickens et al., 2004; Gawron et al., 2002).

Due to lack of evidence regarding these aspects of the schedule of requirements and the concomitant uncertainty, the decision was made to conduct this study in the form of a process simulation that must answer this question (size of the clean area surface and height of the supply bridge). In order to be able to carry out the process simulations successfully, it was necessary to construct a mock-up of the supply bridge. In addition to the primary question whether the dimensions chosen in the design were satisfactory, the study also offered an opportunity to investigate whether the centered positioning of the clean area in the OR was actually the most practical layout when viewed from the logistical processes in the OR. At the time the simulation was conducted, the design had already entered the final design phase.

A survey of the expectations regarding the design among OR experts such as installation experts, a doctor-microbiologist and functional designers, as well as the current users and the policy-makers in the hospitals surgery department was carried out ahead of time. The survey not only looked at the hygiene aspects of the design, but also covered the logistical aspects. The choice for separate preparation rooms connected to the OR resulted in materials and patients entering the clean area through separate routes. This would mean a new logistical process for the hospital that they had not gained any experience with yet. Because of this, the end users adopted a positive, yet critical basic attitude. All of these aspects combined led to the following research questions:

- Are the dimensions (surface area and height) of the clean area large enough to carry out most interventions?

- Is the central position of the clean area in the operating room the most optimal one, when viewed from a logistical processes perspective?

These research questions have been converted into the following hypotheses, which have formed the foundation for the research:

1. The $3 \times 3 \mathrm{~m}^{2}$ clean area surface is large enough to carry out all interventions.

2. Positioning the clean area in the middle of the operating room results in an optimal layout for moving in the patient and equipment in the operating room.

3. The classic position of the anesthesia section, near the entrance to the operating room, is optimal for the logistical processes. 
4. A supply bridge height of 2.05 meters results in the clean area being sufficiently accessible for high medical equipment and reachable for the staff to make connections (electricity, vacuum, etc.) .

\section{Methods \\ Procedure}

The study was conducted through applying observation techniques (Israelski, 2011; Rogers, Patterson, \& Render, 2012; Gosbee \& Gosbee, 2012; Sandberg, 2005). Woods (2003) distinguished three classes of research methods, varying in shaping the conditions for observation: (1) natural history methods, (2) staged world observations using simulated problems, and (3) Spartan lab experiments using experimenter-created artificial tasks. We used staged-world observations, in which we "staged" situations of interest through simulations, in this case representative (or typical) surgical procedures. The relative advantage over natural history methods is that there is more control over the conditions shaping the observations: by controlling the environment and manipulating the scenarios, we will be able to answer our research questions with higher validity than when relying on ethnographic observations of work that occurs in situ. Compared to Spartan lab experiments, the environment is still sufficiently rich to capture representative expert behavior, thus allowing for higher external validity. In particular, our data collection process involved four observers who sequentially captured in detail both (1) observable activities and verbalizations, and (2) self-report data about how the new physical layout supported performance (Rogers, Patterson, \& Render, 2012). For each of the four typical procedures a specialized surgical team that was accustomed to working together on that specific procedure in practice was used. These four surgical teams carrying out the typical procedures were overtly observed in detail. Ethics approval was unnecessary and therefore not obtained, as no real nor simulated patients were involved.

Because the placement of instruments, equipment and persons will be different than in current practice, we decided to simulate these typical operations in an environment that had the spatial characteristics of the new design (size and height of the ultraclean ventilation (UCV) canopy and support bridge). The typical operations were selected with regard to the positioning of the patient and the number of required instruments (instrument tables), the use of special (large or high) medical equipment and the size of the surgical team given the 
setting of a university medical center (research, education, etc.). The simulations were directed by a human factors expert with experience in the analysis of workflows using simulation. His main role was to have those present actually carry out the actions rather than limiting themselves to describing how they thought they would carry out their actions. This is because our perception of our actions may be quite different from the actual action, especially in the case of routine activities to which we have no conscious access (Nisbett \& Wilson, 1977; Wilson, 2002; Anderson, 1987). In doing this, it is necessary to execute actions that belong together consecutively so that the interface between the actions can also be included. The human factors expert therefore also played an important role in determining the time allocated to simulation and the time allocated to discussion. The four observers (including the human factors expert) who watched the actions carried out by the surgical team during the process simulation received forms prior to the simulation on which they could record their observations. These forms were collected and evaluated after the simulation. After completion of the simulation, the observations were discussed with the surgical teams and either a conclusion was drawn based on consensus, or the positioning was adjusted to see if it would lead to a more workable situation. This is in line with the fundamentally observational and discovery oriented nature of staged world studies (Woods, 2003). The simulations of these operations were also recorded on video for more detailed study later (Mackenzie \& Xiao, 2012).

\section{Composition of the Surgical Teams Participating in the Simulations}

The research was carried out with four separate complete surgical teams-surgeon(s), anesthetists, and instrument staff-that were accustomed to working together on that specific intervention in practice. This enabled in depth discussions on the new workflow in the new environment (with a mock-up). It also made it possible to approach the process as realistically as possible in this way, thus enabling sufficient external validity. Table 1 presents the composition of each surgical team. 
Table 1. Composition of surgical team for the simulated interventions.

\begin{tabular}{|c|c|c|c|c|}
\hline $\begin{array}{ll} & \text { Intervention } \\
\text { Surgical team } & \end{array}$ & $\begin{array}{l}\text { Trauma } \\
\text { (hip) }\end{array}$ & $\begin{array}{l}\text { Da Vinci } \\
\text { (surgical } \\
\text { robot) }\end{array}$ & $\begin{array}{l}\text { Trepanation } \\
\text { (using } \\
\text { microscope) }\end{array}$ & $\begin{array}{l}\text { Orthopedic } \\
\text { (knee/hip/laparoscopy) }\end{array}$ \\
\hline Surgeon & 1 & 1 & 1 & 1 \\
\hline Assistant surgeon & 1 & & 1 & \\
\hline Anesthetist & 1 & 1 & 1 & 1 \\
\hline Assistant Anesthetist & 1 & 1 & 1 & 1 \\
\hline Scrub nurse & 1 & 1 & 1 & 1 \\
\hline Staff in the periphery area & 1 & 1 & 1 & 2 \\
\hline Total size of the medical staff & 6 & 5 & 5 & 6 \\
\hline Observers & 4 & 4 & 4 & 4 \\
\hline Process director & 1 & 1 & 1 & 1 \\
\hline
\end{tabular}

\section{Observers}

The observers' task during the process simulation was to watch whether conflicts with the spatial parameters arose during the simulations. Every observer concentrated on one side of the clean area. Prior to the simulation, the observers received a form that showed the initial layout and the boundaries of the clean area. The observers could use these forms to record where conflicts occurred per phase of the simulation (preparation, operation, completion), for example, where people and equipment which should stay sterile crossed the clean area boundaries, where high equipment touched the supply bridge, whether non-ergonomic movements were needed to keep the actions inside the clean area, and other incidents that caught their attention. The forms also provided room for indicating good locations for connecting to compressed air, vacuum and electricity on the supply bridge.

\section{Variables}

The variables recorded concern the number and location of conflicts with regard to crossing the clean area boundaries or to touching the supply bridge. The logistical movements of patient and equipment that occurred were also recorded, including the requirements for maneuvering the equipment well. Each surgical team indicated the best locations for the connections of compressed air, vacuum and electricity on the supply bridge.

\section{Environment}

Because the surface of the clean area and the height of the supply bridge were part of the aspects to be tested, we decided to construct a mock-up of the supply bridge (see Figure 2). The height of this supply bridge could be adjusted from 2.0 to $2.15 \mathrm{~m}$, in $5 \mathrm{~cm}$ increments. This mock-up could also be freely moved around in the operating room. It was also possible 
to use symbols to indicate the desired location for the various connections on the supply bridge. This applied to electrical connections, connections for medical gases and connections for vacuum (extraction). Furthermore, we decided to carry out the tests in the operating department itself by taking one of the operating rooms out of commission for a week. In this way, we gained access to all the equipment that is typically available in the OR. All the process steps, from positioning, induction and operation, through to departure from the operating room, were enacted during the simulation, and their spatial consequences were observed.

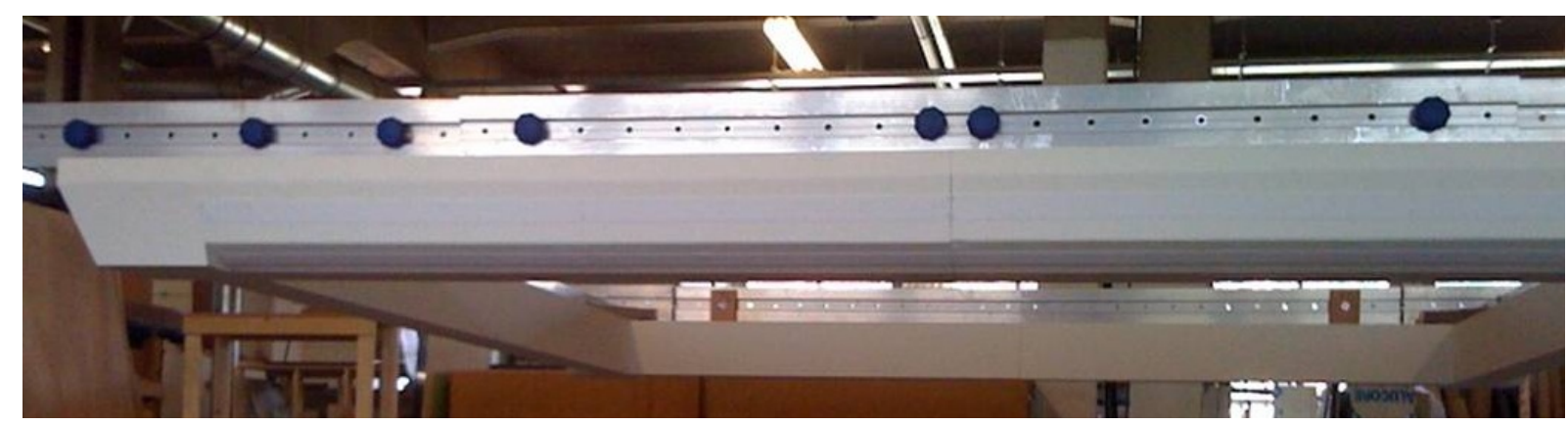

Figure 2: Mock-up being built.

\section{Scenarios}

Typical interventions for testing the hypotheses were determined in consultation with the clinical teams. While taking into account the variables (size of the clean area and the necessary height), the following typical procedures were chosen:

1. Trauma (hip)

2. Da Vinci surgical robot

3. Trepanation (brain surgery using microscope)

4. Orthopedic (knee/hip/laparoscopy)

For each of these typical interventions, we determined in advance which staff of the operating theatre, which equipment, and how many instrument tables were involved in the operation. Drawings of probable set-ups were produced based on this information (Figure 3, 4, 5 and 6).

\section{Trauma (Hip)}

This intervention has typical requirements for the positioning of the patient and the number of required instruments (instrument tables), the use of the mobile imaging equipment (C-arm) 
and the size of the surgical team. Furthermore, the patient is considered to be part of the dirty zone of the operation room during this type of operation, which means specific positioning within the clean area is needed (Figure 3).

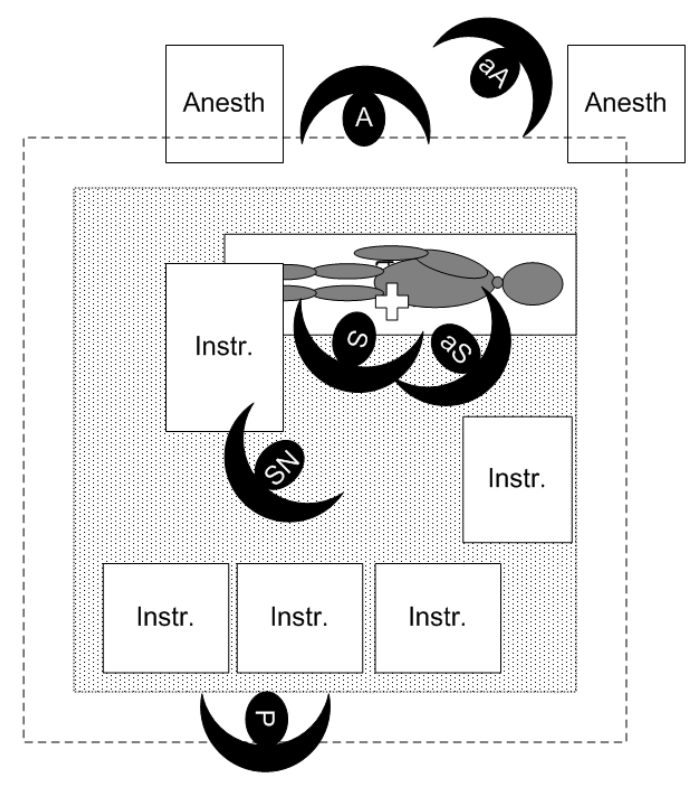

Figure 3: Initial position hip trauma.

\section{Da Vinci Surgical Robot}

This intervention has typical requirements for the space needed for medical equipment (robot and control console) and the height of the supply bridge while maneuvering the equipment (Figure 4).

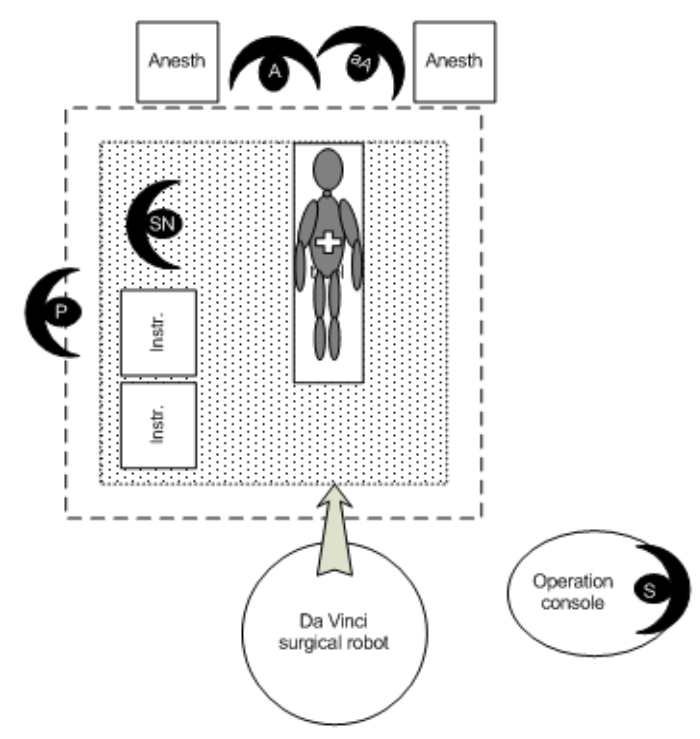

Figure 4: Initial position Da Vinci surgical robot. 


\section{Trepanation}

This intervention has typical requirements for the space needed for medical equipment (microscope) and the height of the supply bridge (Figure 5).

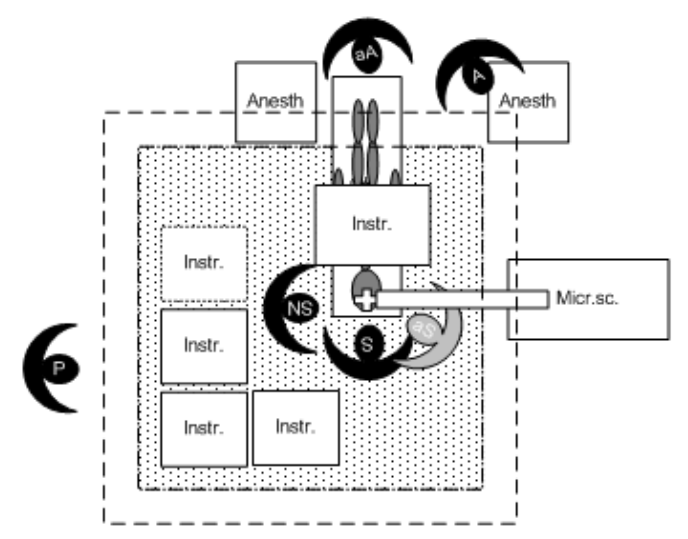

Figure 5: Initial position trepanation with surgical microscope.

\section{Orthopedic}

This intervention has typical requirements with regard to the positioning of the patient and the number of required instruments (instrument tables), the use of the C-arm and the size of the surgical team. Furthermore, orthopedic operations are often characterized by a noncentral positioning of the wound area. In addition, the orthopedic surgeons at this hospital make use of helmets, which results in them being larger than other surgeons and more prone to hit the supply bridge with the helmet (Figure 6).

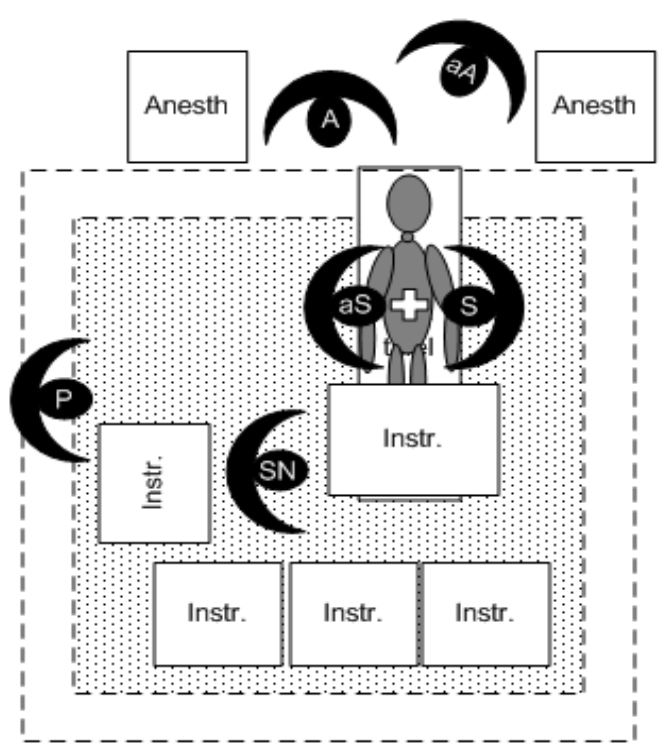

Figure 6: Initial position orthopedic intervention. 


\section{Results}

\section{Hypothesis 1: The Dimensions of the Clean Area of $3 \times 3 \mathrm{M}^{2}$ Are Large Enough to Carry Out}

All Interventions

The lack of conflicts with the boundaries of the clean area and the height of the supply bridge during the simulations shows that A 3.0 X 3.0 M² LARGE CLEAN AREA UNDER THE UNIDIRECTIONAL DOWN-FLOW PLENUM IS LARGE ENOUGH TO SATISFACTORILY POSITION THE PATIENT, THE SURGICAL TEAM, AND THE STERILE INSTRUMENTS REQUIRED DURING THE FOUR INTERVENTIONS THAT WERE SIMULATED. However, an important prerequisite for this to be true is that a movable operation table be used. The space that the instrument staff and instrument tables occupy on one side of the table, is more than half of the clean area (see also Figure 1, above). IF A FIXED OPERATION TABLE IS USED, THE SURFACE OF THE CLEAN AREA WILL NEED TO BE LARGER. The dimensions required in this situation have not been researched as part of this study. The positioning of the instruments relative to the table is also so diverse for the various operations that a fixed non-central position for the operation table does not offer a solution either.

Discussing these dimensions with the team after the process simulation showed that bottlenecks may arise during operations of the "multiple trauma” type. Although this kind of operation was not simulated, it could be deduced from the various set-ups that if multiple surgical teams were operating on a patient simultaneously, it would become difficult to position the whole wound area, all the instruments and all the staff in the clean area, straight under the unidirectional air flow terminal together. For this reason, 3 out of 19 operating rooms in the design at hand will be fitted with a larger unidirectional airflow terminal measuring $3.20 \times 3.20 \mathrm{~m}^{2}$.

By drawing the set-up it can be determined that these dimensions would offer just enough space for a double team. Even larger dimensions have not been considered for budgetary and technical reasons. Needless to say, these larger dimensions do not form an obstacle for the other operations, thus resulting in maximum flexibility (every OR can be used for every type of intervention, excluding the multiple trauma). 


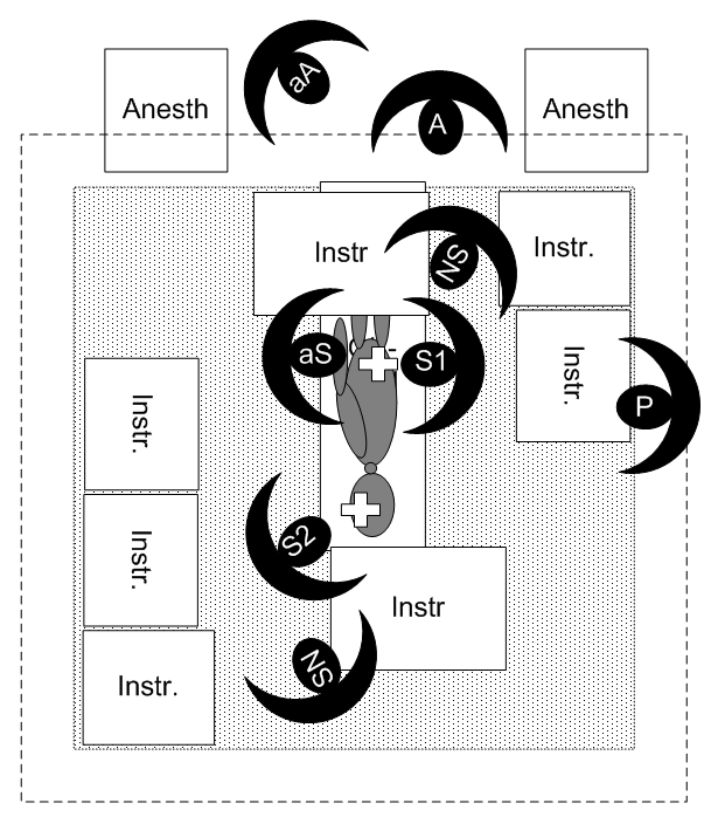

Figure 7: Double surgical team set-up under a 3.2 x $3.2 \mathrm{~m}^{2}$ plenum.

Our simulations showed that the hypothesis is true given the use of a movable operation table and excluding multi trauma settings.

Hypothesis 2: Positioning the Clean Area in the Middle of the Operating Room Results in an Optimal Logistical Layout of the Operating Room

During the process simulations it was shown that, in order to be able to maneuver and position a C-arm well, it is necessary to have at least $1.75 \mathrm{~m}$ of free space between the clean area and the walls of the operating room. After taking an X-ray, the C-arm was positioned outside of the clean area again. A minimum of $2.25 \mathrm{~m}$ was needed for the positioning of the anesthesia staff and the required equipment (crash cart, cart with materials). For this reason, we conclude that there should be a minimum space of $2.25 \mathrm{~m}$ between the clean area and the walls. Based on the discussion with the staff and medics and the preformed simulations we concluded that THE REMAINING SPACE INSIDE THE OR SHOULD PREFERABLY BE SITUATED TOGETHER ON THE CORRIDOR SIDE, RATHER THAN BEING EQUALLY DIVIDED AROUND THE CLEAN AREA (see Figure 8). In the case of larger dimensions of the operating room the unidirectional down-flow terminal (ultraclean ventilation (UCV) canopy) is not positioned centrally in the operating room as is customary in traditional designs. It can also be deduced from this that the minimum size of an operating 
room that is suitable for all types of operations, with a unidirectional down-flow plenum (ultraclean ventilation (UCV) canopy) measuring $3.0 \times 3.0 \mathrm{~m}$ and with good logistics is $7.5 \mathrm{x}$ $7.5=56.25 \mathrm{~m}^{2}$. This size includes the space for the periphery area and the temporary storage of equipment in the operating room. The hypothesis must thus be rejected: The non-central set-up of the plenum (in the case of larger ORs) offers a better logistical layout of the operating room.
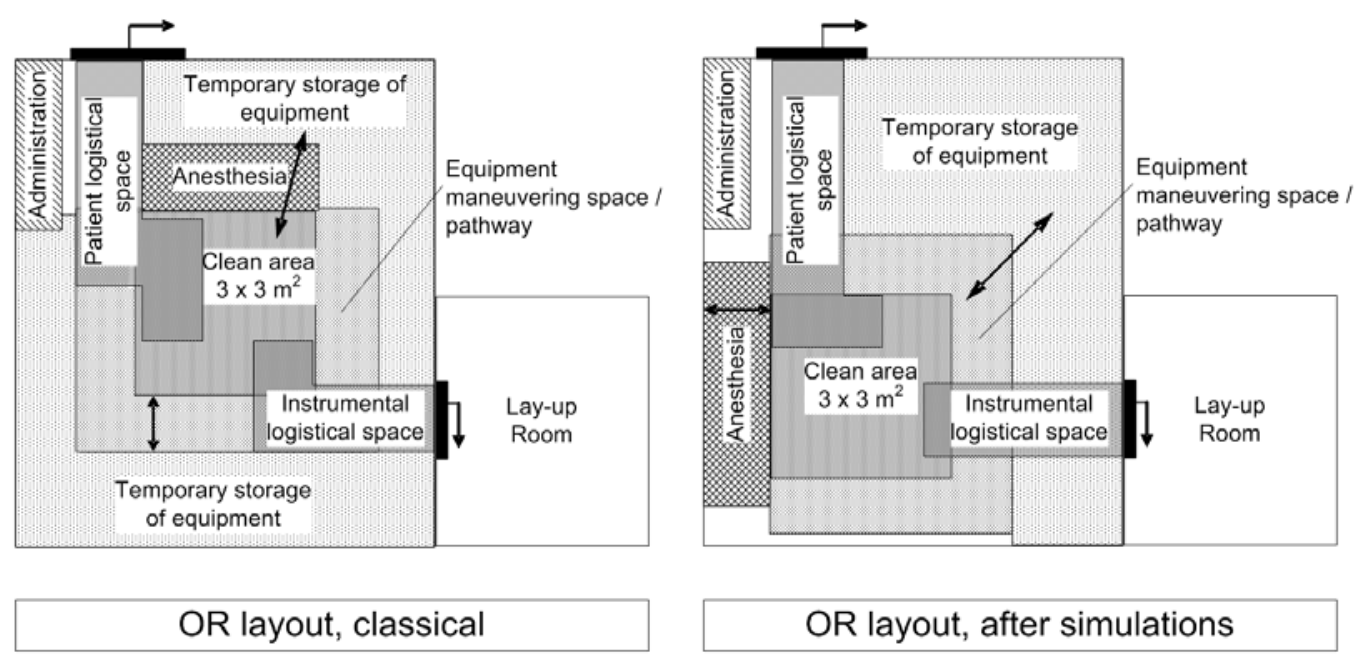

Figure 8: OR layout before and after the simulations.

\section{Hypothesis 3: The Classic Position of the Anesthesia Section in the Operating Room Is Optimal for the Logistical Processes}

During the process simulations, it became apparent that the classic position of the anesthesia section (with the back facing the corridor) hinders the transport of the patient's bed from the corridor and position it alongside the operating table without a lot of maneuvering. It was also shown that the risk of bumping into the anesthetic equipment with the bed was high. One outcome of the discussions with the surgical team following the process simulations was that there were no objections to moving the anesthetists' section to the side of the OR. This creates better access to the clean area, both for the patient and for the instruments and equipment (see also Figure 8). The hypothesis must thus be rejected: THE POSITIONING OF THE ANESTHESIA SECTION ON THE SIDE OF THE CLEAN AREA OFFERS A BETTER LOGISTICAL LAYOUT OF THE OPERATING ROOM THAN THE TRADITIONAL SET-UP BETWEEN THE CLEAN AREA AND THE ENTRANCE. 


\section{Hypothesis 4: A Supply Bridge Height of 2.05 Meters Results in the Clean Area Being Sufficiently Accessible to High Medical Equipment}

The scenarios with the surgical microscope and the Da Vinci surgical robot led to conflicts with the supply bridge height. The Da Vinci surgical robot is positioned over the patient with its arms in the highest possible position. Part of the current routine is to put the sterile covers on the arms of the robot outside of the clean area, after which the robot is rolled into the clean area with its arms in the highest position and then placed over the patient. Execution of this common procedure in this manner was shown to be impossible if the supply bridge is $2.05 \mathrm{~m}$ high. However, during the use of the Da Vinci robot, the clean area is so empty (because of the absence of tables, instruments and staff) that there is sufficient space to extend the robot to its highest position after it is under the unidirectional down flow terminal and to subsequently maneuver it over the patient.

During the trepanation, the arm of the current surgical microscope touched the supply bridge when it was positioned next to the patient (see Figure 5). It was possible to avoid the conflict by positioning the microscope foot diagonally behind the surgeon and having the microscope arm come over the shoulder of the surgeon. This set-up is workable for the surgeon, albeit less pleasant.

The chance of this type of conflict occurring is largely dependent on the type of microscope arm. An arm in which the highest point of the lower movable part of the arm does not get any higher than 2.0 meters does not conflict with the supply bridge. The current microscope arm reaches up to 2.08 meters, $3 \mathrm{~cm}$ higher than the bottom edge of the supply bridge.

During operations involving an operation helmet, such as in orthopedics, there may be a conflict with the height if the surgeons are tall. This actually only occurs when entering and exiting the clean area. During the operation, the members of the surgical team are inside the supply bridge area and thus under the unidirectional down flow terminal.

For many staff in the periphery area, a height of 2.05 meters is workable for making connections in the slanted part of the supply bridge (compressed air, electricity, vacuum, etc.). For shorter people $(<1.65 \mathrm{~m})$ the supply bridge needs to be lower for these activities to 
be carried out in an ergonomically sound way. A lower height would lead to a marked increase in the number of conflicts. It is therefore suggested that shorter people could make use of a portable step.

In summary, the process simulations showed that because of the height of both the arm of the current microscope and the surgical robot, the supply bridge will need to either be roughly 10 cm higher, U-shaped, or wider so that the whole microscope fits into the sterile zone. However, these solutions have the following drawbacks:

- A higher supply bridge would be more difficult to reach for plugging in equipment and unplugging it.

- When a three-sided U-shaped supply bridge is used, it is more complicated to change the equipment set-up to a mirrored set-up and there is less space for making connections on the supply bridge. A three-sided U-shaped supply bridge is seen to be a lesser solution. In order to keep the release point of the laminar airflow as low as possible, it is desirable to have the supply bridge connect (seamlessly) with the plenum side screen. The fourth side of the supply bridge also turns out to be indispensable for some of the many connection points needed. Besides, if the supply bridge were U-shaped, it would only be possible to move equipment hanging on the bridge to the other side of the bridge by way of the anesthesia side of the bridge, which will itself be full of connections and anesthesia equipment already.

- A wider bridge to allow more "arm space" for the microscope means the bridge no longer fits the surface of the unidirectional down flow terminal that the whole terminal must be made larger. If the bridge does not connect with the plenum, the risks of a poorly directed airflow increase, resulting in possible turbulence along the edges of the down-flow. This results in a substantial decrease in the size of the sterile zone. A (standard) larger plenum means more air needs to be blown in, resulting in greater energy usage and a more expensive plenum.

Taking all these factors into consideration, THE 2.05 M HEIGHT OF THE SUPPLY BRIDGE IS A GOOD COMPROMISE. During the discussion with the surgical team after the process simulation, it was therefore stated that a square, closed supply bridge at a height 
of $2.05 \mathrm{~m}$ from the floor and with internal dimensions of $3.0 \times 3.0 \mathrm{~m}^{2}$ would be the starting point for the future routines and for the purchase of equipment within the hospital. For the robot, this means that the arms will only be moved to the highest position after it has been rolled under the bridge. The simulation showed that there is ample space in the clean area to be able to carry out this maneuver safely. For the microscope, it means that the microscopes to be purchased in the future will be partly selected based on the maximum height of the part of the arm that passes under the bridge.

Taking the aforementioned restrictions into consideration, we can thus state that hypothesis 4 need not be rejected and that a height of 2.05 meters does not lead to (insurmountable) conflicts for the accessibility of the clean area with equipment from the pathway.

The layout that has resulted from the process simulations (Figure 8, above) differs on essential aspects from the layout that was the result of the traditional design process (see Figure 1, above). The main difference is the positioning of the clean area in the operating room (non-central set-up versus central set-up) and the positioning of the anesthesia section. This has resulted in the modification of the original final design that was arrived at through a traditional design process, to a design based on the results of the process simulation (see Figure 8).

\section{Discussion}

The objective of this study was to evaluate a traditionally designed operating room using simulation of various surgical workflows. The primary research question was whether the clean area under the unidirectional air flow terminal would be large enough to enable smooth, unhindered, task performance, while at the same time not being too far away to reach it with the equipment connections. In addition to the primary question, the study also offered an opportunity to investigate the logistical consequences of the centered positioning of the clean area in the OR. The results clearly showed that a clean area measuring 3 x 3 meters and a supply bridge height of 2.05 meters was satisfactory for most situations, provided a movable operation table was used. The only cases in which conflicts with the supply bridge were observed were during the use of a surgical robot (Da Vinci) and a surgical microscope. During multiple trauma interventions, bottlenecks regarding the dimensions of the clean area will probably arise. 
Due to the choice for a supply bridge with a side screen around the unidirectional down flow terminal, it is assumed that the unidirectional airflow will create a strong division between the clean area and the environment covering the whole surface. After the airflow is no longer directed by the side screen, some mixture of the air with the environment air will take place along the edge of the clean area. This so-called constriction of the air column will in practice reduce the size of the clean area. However, during the process simulations, it was assumed that the plenum surface projected on the floor, determined by the inner dimensions of the side screens, represented the clean area. This position can be defended because the reduction of clean area due to the side screens is presumably very limited at a height of roughly 1.10 meters above the floor, the height at which the intervention is executed.

With respect to the height of the supply bridge, one more thing to mention is that the orientation of the surfaces that the connections for the medical gases, earth points and vacuum are situated in has an influence on the workable free height of the bridge. A person with a given length can still plug in a gas tube at a certain height if it has been turned toward the person in an ergonomic manner. It is easier to plug the tube in at a certain height if it is done diagonally from below, rather than from a position that is parallel to the floor. These matters, however, require additional research to determine what the best orientations and positions of the various connections are.

It is also noted that the simulations made it clear that the accessibility of the operating area with equipment like the C-arm, and the lines of vision in the operating room, have an influence on the ergonomic layout of the supply bridge. This is because the equipment wires and tubes hinder the access to the clean area. Both aspects force the connections towards the corners of the bridge.

During process simulations, there is always the issue of whether to study current workflows or future (new) workflows that are better adjusted to the new situation. In this study, the choice was made to base the process simulation on the existing routines in order to prevent the teams from being too self-conscious about how they worked. If there had been more time for preparation, including training on the new future workflow, it would have been possible to have a more compact set-up in some instances. However, the means for such an approach 
were lacking, e.g., for freeing up operating rooms and surgical teams for instruction and training.

In addition to testing the hypotheses, this study also offered the researchers an opportunity to find out whether process simulation is a useable tool in the design process and whether it creates added value. The method is more expensive than a traditional paper design because it requires the construction of a mock-up, the input of staff and the use of an operating room for one week. Besides, it requests more time due to the necessary preparation.

Process simulations also have made an important contribution to the insights medical personnel has regarding the design and future routines with separate preparation rooms and a unidirectional down-flow system. This has resulted in greater support for the design. The added value of a process simulation versus the expert opinions that form the basis for the traditional design process, is that issues that reside in peoples' perceptions can be objectivized through observation of the process simulation. People often think that things happen differently than what is shown to happen through the actions they perform. Basic actions can also, unconsciously, be considered to be unimportant while they actually do have an impact on the space. It is difficult to determine how much space an action takes without actually trying it. This is an essential question for an operating room that has a relatively limited sterile area. The added value of the process simulation was mainly in the following areas:

- Spatial mapping of dynamic processes

- Determination of required free (logistical) periphery area and lines of sight

- The objectivization of perceptions

- The creation of support for innovative designs

The hospital's project team has found the influence of the process simulations on the design to be considerable, because of their influence on the basic layout and technical installations. Because changes later on in the design process always have concomitant higher costs, it is necessary for the process simulations to take place early on in the design process, as early as the design brief or schematic design phase. 


\section{Conclusions}

Process simulation can have significant impact on the final design and is a very suitable method to support the design process. The process simulation of four typical interventions has led to significantly different operating room layouts than were arrived at through the traditional design process. The main difference is the positioning of the clean area in the operating room (non-central set-up versus central set-up) and the positioning of the anesthesia section.

The results clearly showed that a clean area measuring 3 x 3 meters and a supply bridge height of 2.05 meters was satisfactory for most situations, provided a movable operation table was used.

Success factors for this type of process simulation have shown to be:

- Process simulations should to take place early on in the design process, as early as the design brief or schematic design phase.

- The more realistic a situation is, the more accurate the actions will be. In other words, make as much use of the actual people and instruments / equipment involved in the simulations as possible, and make sure the spatial characteristics of the simulation are the same as those of the design.

- Ensure that the variables being investigated can actually be varied during the simulations. For instance, movable walls to vary the size of the space and the possibility of changing the position of the operating table within the clean area.

- Ensure that all relevant staff members and medics are present during the entire simulation.

- Make sure there is someone in charge who monitors the process and leads the discussions.

- Make use of independent observers that have no other role during the simulations and the discussions.

- The clearer the working instructions are, the less discussion there will be about what to do, but make sure people are free to act naturally.

- Go through as many connected actions as possible, but offer space for discussion after a (partial) session. 


\section{Implications for Practice}

- This study demonstrates that process simulation can have significant impact on the final design and is a very suitable method to support the design process.

- Process simulation can play an important role in Evidence Based Design.

- There are success factors that should be taken into account using process simulation as a design instrument.

\section{References}

Anderson, J.R. (1987). Skill acquisition: Compilation of weak-method problem situations. Psychological Review, 94, 192-210.

Baumgart, A., Denz, C., Bender, H.-J., \& Schleppers, A. (2009). How work context affects operating room processes: using data mining and computer simulation to analyze facility and process design. Quality Management in Health Care, 18(4), 205-314.

Bittermann, M. S. (2009). Intelligent Design Objects (IDO): A cognitive approach for performance-based design. PhD thesis, Delft University of Technology, The Netherlands.

Boxel, van, E., Koreman, K., Verweij, M., Rodermond, J., Huijsman R., Hansen, B., Schaap, P. M. (2007). Bouwen aan de Architectuur van de zorg (Building Health Care Architecture). ISBN-978-90-811772-1-4. Retrieved from www.architectureinhealth.nl

Chen, X., Xu, B.N., Meng, X., Zhang, J., Yu, X., \& Zhou, D. (2011). Dual-room 1.5-T intraoperative magnetic resonance imaging suite with a movable magnet: implementation and preliminary experience. Neurosurgical Review, 1-16, article in press.

Dunston, P.S., Arns, L.L., \& McGlothlin, J.D. (2007). An immersive virtual reality mock-up for design review of hospital patient rooms. 7th International Conference on Construction Applications of Virtual Reality, October 22-23.

Gawron, V. J., Dennison, T. W., \& Biferno, M. A. (2002). Mock-ups, models, simulations and embedded testing. Handbook of Human Factors Testing and Evaluation, Second Edition, Eds.: Charlton, S.G. \& O’Brien, T.G.; Lawrence Erlbaum Associates, Inc., Publishers, Mahwah, New Jersey, London.

Gosbee, J., \& Gosbee, L.L. (2012). Usability evaluation in health care. Handbook of Human Factors and Ergonomics in Health Care and Patient Safety, Second Edition, Ed.: Carayon, P.; CRC Press, Boca Raton, FL. 
Israelski, E.W. (2011). Testing and evaluation. Handbook of Human Factors in Medical Device Design, Eds.: Weinger, M.B., Wiklund, M.E., and Gardner-Bonneau, D.J.; CRC Press, Boca Raton, FL.

Mackenzie, C.F., \& Xiao, Y. (2012). Video analysis: An approach for use in health care. Handbook of Human Factors and Ergonomics in Health Care and Patient Safety, Second Edition, Ed.: Carayon, P.; CRC Press, Boca Raton, FL.

Moorthy, K., Munz, Y., Undre, S., \& Darzi, A. (2004). Objective evaluation of the effect of noise on the performance of a complex laparoscopic task. Surgery, 136(1), 25-30.

Nisbett, R.E., \& Wilson, T.D. (1977). Telling more than we can know: Verbal reports on mental processes. Psychological Review, 84(3), 231-259.

Peavey, E., Zoss, J., Watkins, N. (2012). Simulation and mock-up research methods to enhance design decision-making. Health Environments Research \& Design Journal, 5(3), 133-144.

Reiling, J.G., Knutzen, B.L., Wallen, T.K., McCullough, S., Miller, R. \& Chernos, S. (2004). Patient safety: Enhancing the traditional hospital design process: A focus on patient safety. Joint Commission Journal on Quality and Safety, 30(3).

Rogers, M.L., Patterson, E.S., \& Render, M.L. (2012). Cognitive work analysis in health care. Handbook of Human Factors and Ergonomics in Health Care and Patient Safety, Second Edition, Ed.: Carayon, P.; CRC Press, Boca Raton, FL.

Sadler, B.L., Berry, L.L., Guenther, R., Hamilton, K., Hessler, F.A., Merritt, C. \& Parker, D. (2011). Fable hospital 2.0: The business case for building better healthcare facilities. Hasting Center Report 41(1), 13-23.

Sandberg, W.S., Daily, B., Egan, M., Stahl, J.E., Goldman, J.M., Wiklund, R.A. \& Rattner, D. (2005). Deliberate perioperative systems design improves operating room throughput. Anaesthesiology, 103(2), 406-418.

Sanderson, P.M., Tosh, N., Philp, S., Rudie, J., Watson, M.O., \& Russell, W.J. (2005). The effects of ambient music on simulated anaesthesia monitoring. Anaesthesia, 60(11), 10731078.

Sehulster, L.M., Chinn, R.Y.W., Arduino, M.J., Carpenter, J., Donlan, R., Ashford, D., Cleveland J. (2004). Guidelines for environmental infection control in health-care facilities. Recommendations from CDC and the Healthcare Infection Control Practices Advisory Committee (HICPAC). Chicago, IL: American Society for Healthcare Engineering/American Hospital Association. 
Strauss, G., Aries, F., Abri, O., Dietz, A., Meixensberger, J., \& Lüth, T. (2010). Conception, realization and analysis of a modern operating theatre workplace for ENT surgery. HNO 58(11), 1074-1084.

Suzuki, T. (2010). Cleanliness in the operating room. Japanese Journal of Anesthesiology, 59(5), 556-563.

Ulrich, R.S., Zimring, C., Zhu, X., DuBose, J., Seo, H.-B., Choi, Y.-S., Quan, X. and Joseph, A. (2008). A review of the research literature on evidence-based healthcare design, Health Environment Research and Design, 1(3),101-165.

Wickens, C.D., Lee, J.D., Liu, Y., \& Gordon Becker, S.E. (2004). An introduction to Human Factors Engineering (2nd ed.). Upper Saddle River, NJ: Pearson Education, Inc.

Wilson, T.D. (2002). Strangers to ourselves: Discovering the adaptive unconscious. Cambridge, MA: The Belknap Press of Harvard University Press.

Woods, D.D. (2003). Discovering how distributed cognitive systems work. Handbook of Cognitive Task Design, Ed.: Hollnagel, E.; Lawrence Erlbaum Associates, Publishers, Mahwah, NJ.

Yavuz, S.S., Bicer, Y., Yapici, N., Kalaca, S., Aydin, O.O., Camur, G. et al. (2006). Analysis of risk factors for sternal surgical site infection: Emphasizing the appropriate ventilation of operating theaters. Infection Control and Hospital Epidemiology, 27(9), 958-963. 